\title{
Method of Moments Based Modelling of Race-Track Fluxgate Sensitivity Dependence on Track and Core Width
}

\begin{abstract}
A. Ostaszewska-LiżewskA* And R. SzewCZYK
Warsaw University of Technology, Faculty of Mechatronics, sw. A. Boboli 8, 02-525 Warsaw, Poland

Intensive development of thin-layer based fluxgate sensors produced as printed circuit boards demand proper tools for modelling their functional characteristics. This paper presents the application of the method of moments utilizing uniform square meshes to avoid problems with thin layer modelling utilizing finite elements method. Exemplary results of modelling indicate that the sensitivity of race-track shaped fluxgate sensor strongly depends on the width of the track, whereas the influence of the width of the core on the fluxgate sensitivity is about $3 \%$. This information is important for development of printed circuit boards based fluxgate sensors with racetrack-shaped cores, as it can lead to significant reduction of the sensor's perpendicular dimension.
\end{abstract}

DOI: 10.12693/APhysPolA.137.697

PACS/topics: method of moments, magnetic modelling, fluxgate sensor

\section{Introduction}

Since its introduction in 1936, fluxgate sensors are commonly used in the precision magnetic field measurements. Such sensors are reliable, robust, and exhibit resolution up to $10 \mathrm{pT}$ operating in the room temperature [1]. On the other hand, typical fluxgate sensors are not suitable for miniaturization and they require sophisticated electronic signal processing circuits [2]. For this reason, during last few years, intensive efforts were taken to develop miniature fluxgate sensors (such as DRV 425 of Texas Instruments) suitable for scientific and industrial applications.

Recent developments indicate that the most promising direction of fluxgate sensors development are sensors with thin layer core (e.g., made of amorphous alloy ribbon [3] etched to specific shape) with driving and sensing winding made as tracks on multilayer printed board circuit (PCB) $[4,5]$.

Intensive development of thin layer based fluxgate sensors created needs for tools for modelling their characteristics. Such models are necessary for optimization of the shape of the core of fluxgate sensors, considering demagnetization factor [6,7], as well as constrains created by sensor geometry and windings requirements. For such modelling, the finite element method (FEM) is commonly used [8]. However, in the case of thin film devices, number of elements required for modelling increases radically with decrease in layer thickness [9].

For this reason, alternative methods of modelling the thin layer magnetostatic systems are developed. One

\footnotetext{
* corresponding author; e-mail a.ostaszewska@mchtr.pw.edu.pl
}

of the most promising solutions is based on the methods of moments (MoM) concept introduced by Harrington in 1993 [10]. The method of moments is based on analysis of the influence of hypothetical magnetic moments [11] assigned to each element's border. It should be stressed that the method of moments requires solving the linear equations, which is very efficient in modern, vectorcalculation oriented computers. In addition, recently presented generalization of the MoM for magnetic thin layers with rectangular grid [12] is especially useful for optimization of cores of thin layer sensors.

This paper presents the principles of practical application of the method of moments and the results of optimization of race-track shaped core [13] for fluxgates produced in the PCB-based technology. Moreover, software for this optimization is available as open-source at [14] for further validation and development.

\section{The method of modelling}

The principles of operation of the fluxgate sensor with race-track shaped core are similar to principles of operation of the fluxgate with ring-shaped core presented in [4]. The geometry of the racetrack-shaped core of fluxgate sensor is determined by three parameters: $a$ - half-length of the core, $b$ - width of the core, and $c$ - core's radius. Moreover, a thickness $g$ of the core, as well as material relative permeability $\mu$, determine its demagnetization factor from the point of view of measured magnetic field applied in the direction of core's length. Schematic view of the shape of racetrack-shaped core of fluxgate sensor and its parameters is presented in Fig. 1a, whereas the schematic view of fluxgate sensor in $\mathrm{PCB}$ technology is presented in Fig. 1b.

In the present investigation, the relative magnetic permeability $\mu$ of the core was 1000 , whereas the thickness $g$ of the core was $35 \mu \mathrm{m}$. Such parameters are 
(a)

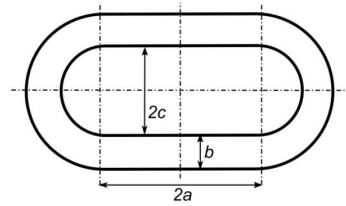

(b)

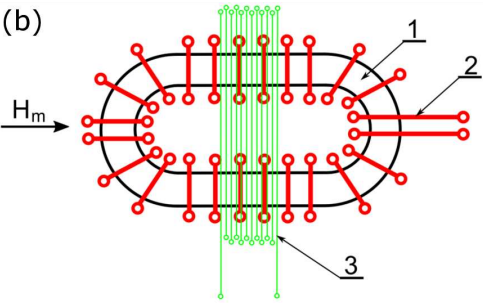

Fig. 1. Schematic view of the fluxgate sensor with racetrack-shaped core: (a) the geometry of the racetrack-shaped core (determined by the parameters $a, b$, and $c$ ), (b) the schematic view of fluxgate sensor in PCB technology: (1) race-track shaped core made of magnetic material, (2) magnetizing winding, (3) sensing winding.

typical for the thin layer cores made of amorphous alloys in as-quenched state [15] developed for fluxgate sensor applications.

The magnetic field distribution in the core was calculated by MoM presented in detail in [16]. On the basis of results of calculation, the influence of the magnetization from external field $H_{m}$ on the output signal of PCB fluxgate sensor was calculated. Due to the linear relative magnetic permeability $\mu$ approximation, only demagnetization factor of race-track core was considered for nearly-linear part of core's characteristic. Schematic view of such sensor is presented in Fig. 1b, whereas the principles of fluxgate sensor operation were described previously [17]. Calculations were carried out with the assumption that sensing winding length in the centre of the core is equal to $2 a / 3$, where $a$ is equal to $15 \mathrm{~mm}$.

\section{Results}

Figure 2a presents the racetrack-shaped core of fluxgate sensor. The core was divided into the square-shaped mesh. Figure $2 \mathrm{~b}$ and $\mathrm{c}$ presents the magnetic field distribution in $x$ and $y$ directions due to the magnetization by external magnetic field in $x$ direction, $H_{m}=100 \mathrm{~A} / \mathrm{min}$. In the presented case, quite high demagnetization can be observed in the core. However, in the $x$ direction (the direction of magnetizing field $H_{x}$ ), the distribution of flux density $B_{x}$ is nearly uniform in the area of race-track shaped core covered by sensing winding. This phenomenon simplifies the calculation of sensitivity of fluxgate sensor. Moreover, results of modelling indicate, that in the present case, maximal value of flux density $B$ in the $y$ direction is about five times lower than in $x$ direction.

On the basis of the result of integration of flux density $B_{x}$ in the part of racetrack-shaped core of fluxgate sensor covered by sensing winding (as it is presented in Fig. 2b), value of output signal from fluxgate was estimated. Figure 3 presents this value as the function of $b$ and $c$ parameters determining the track and core's width respectively (presented in Fig. 1a, where $a$ is equal to $20 \mathrm{~mm}$ ).

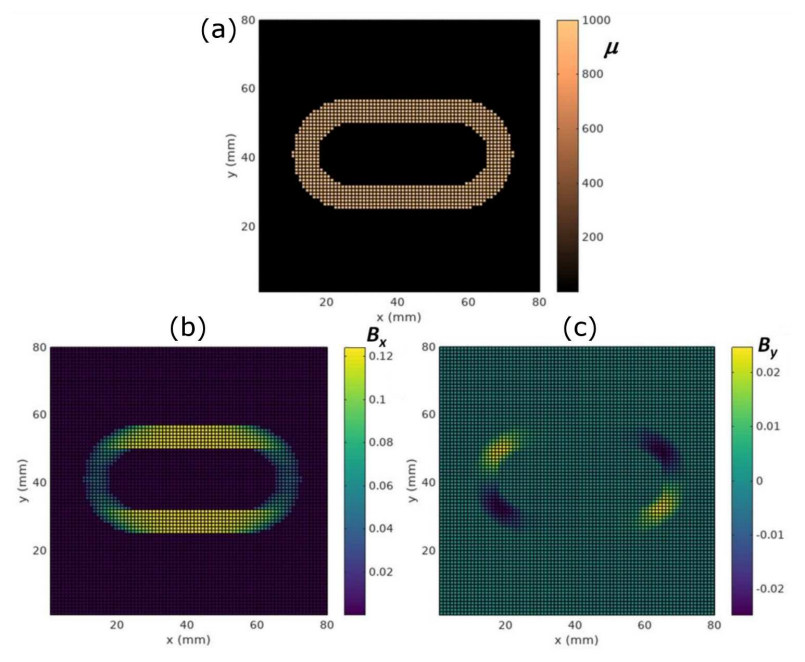

Fig. 2. Racetrack-shaped core of fluxgate sensor: (a) spatial distribution of relative magnetic permeability $\mu$ in the core, (b) the distribution of flux density $B_{x}$ in the core in $x$ direction, (c) the distribution of flux density $B_{y}$ in the core in $y$ direction (both for magnetizing field $\left.H_{x}=100 \mathrm{~A} / \mathrm{m}\right)$.

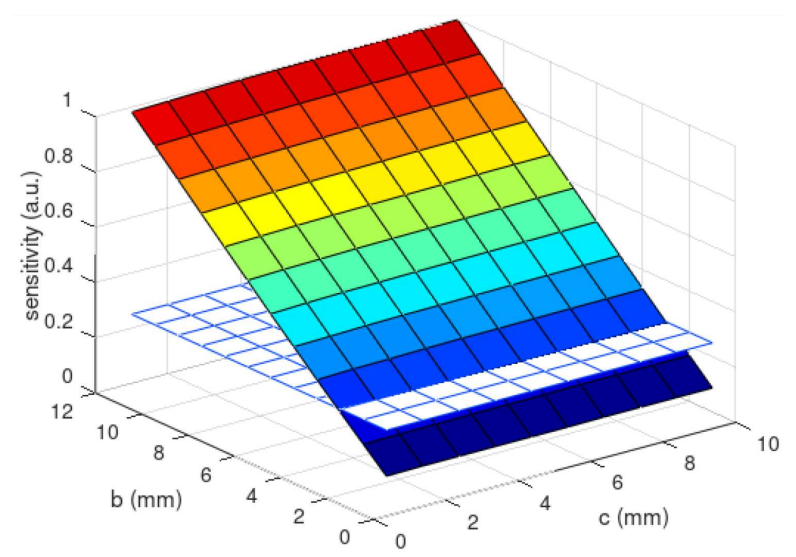

Fig. 3. The dependence of sensitivity of fluxgate sensor with racetrack-shaped core on width $b$ and radius $c$ of the core $(a=20 \mathrm{~mm})$. Blue lined surface indicates minimal expected level of sensitivity of fluxgate sensor for industrial application.

Minimal expected level of sensitivity of fluxgate sensor made in PCB is marked in Fig. 3 as the blue lined surface. The minimal expected level of sensitivity was determined previously during the experimental development of square frame-shaped fluxgate sensors [18].

\section{Conclusions}

It should be highlighted that the approach based on the method of moments utilizing uniform mesh opens new possibilities of modelling thin-film magnetic devices, such as magnetic fluxgates, concentrators, etc. Optimization of the shape of these devices was not possible on the basis of FEM. 
The results of this modelling indicate that the sensitivity of race-track shaped fluxgate sensor strongly depends on the width of the track (parameter $b$ ). On the other hand, the influence of the width of the core (given by parameter $c$ ) on the fluxgate sensitivity is about $3 \%$. As a result it can be neglected from a practical point of view. This information is very important for development of PCB-based fluxgate sensors with racetrack-shaped cores, as it can lead to significant reduction of the sensor's perpendicular dimension.

\section{Acknowledgments}

This work was fully supported by the statutory founds of Institute of Metrology and Biomedical Engineering, Warsaw University of Technology.

\section{References}

[1] P. Ripka, Magnetic Sensors and Magnetometers, Artech 2001.

[2] P. Ripka, Sens. Actuat. A 106, 8 (2003).

[3] S. Tumanski, Electr. Rev. 4, 1 (2010).

[4] A. Tipek, P. Ripka, T. O'Donnell, J. Kubik, Sens. Actuat. A 115, 286 (2004).

[5] J. Kubík, in: Printed Circuit Board Fluxgate Sensors, Ed. P. Ripka, Vol. 1, Shaker Verlag, 2009.
[6] F. Primdahl, B. Hernando, O.V. Nielsen, J.R. Petersen, J. Phys. E Sci. Instrum. 22, 1004 (1989).

[7] J. Kubík, P. Ripka, Sens. Actuat. A Phys. 143, 237 (2008).

[8] J. Kubík, M. Janošek, P. Ripka, J. Electr. Eng. 57, 80 (2006).

[9] R. Szewczyk, "Thin Layer Oriented Magnetostatic Calculation Module for ELMER FEM, Based on the Method of the Moments" in: 22th Int. Conf. on Applied Physics of Condensed Matter, Štrbské Pleso (Slovakia), 2016.

[10] R.F. Harrington, Field Computation by Moment Methods, IEEE Press, 1993.

[11] C. Kittel, Introduction to Solid State Physics, Wiley, 2005.

[12] R. Szewczyk, Acta Phys. Pol. A 131, 845 (2017).

[13] J. Kubik, L. Pavel, P. Ripka, Sens. Actuat. A 130-131, 184 (2006).

[14] R. Szewczyk, Racetrack Fluxgate Optimization.

[15] R. Chamboll, H. Warlimont, IEEE Trans. Magn. 17, 3053 (1981)

[16] R. Szewczyk, Magnetostatic Modelling of Thin Layers Using the Method of Moments and Its Implementation in OCTAVE/MATLAB, Springer, 2018.

[17] S. Tumański, Electr. Rev. 10, 1 (2013).

[18] P. Frydrych, R. Szewczyk, J. Salach, K. Trzcinka, IEEE Trans. Magn. 48, 1485 (2012). 